\title{
SAR MONITORING OF SUBURBAN AREAS BASED ON AN ELECTROMAGNETIC SCATTERING MODEL
}

\author{
Raffaella Guida ${ }^{1}$, Antonio Iodice ${ }^{2}$, Daniele Riccio $^{2}$ \\ ${ }^{1}$ Surrey Space Center \\ University of Surrey \\ Guildford, Surrey \\ Tel. +(44) (0)1483 682227, fax +(44) (0)1483 689503 \\ E-mail: R.Guida@surrey.ac.uk \\ ${ }^{2}$ Dipartimento di Ingegneria Biomedica, Elettronica e delle Telecomunicazioni, \\ Università di Napoli "Federico II" \\ Via Claudio 21, 80125 Napoli, Italy. \\ Tel. +(39)-0817683114, fax +(39)-0815934448 \\ E-mail: \{iodice, daniele.riccio\}@unina.it
}

\begin{abstract}
Cylindrical-shape tanks are typical of any suburban area and often contain dangerous gases or fluids. In this paper, we suggest a way to monitor them by means of high resolution Synthetic Aperture Radar (SAR) images and a scattering model able to quantitatively consider how the radar signal interacts with this kind of structures and how they appear in the SAR images. Adopting the model, geometrical information as the tank height is retrieved from the SAR images in a non-conventional way that is exploiting the information content contained in the double reflection contribution to the radar cross section. Results are compared with more traditional methods and discussed.
\end{abstract}

Index Terms - Synthetic Aperture Radar (SAR), feature extraction, urban areas.

\section{INTRODUCTION}

Inhabited areas and their neighborhoods need persistent surveillance, especially where natural conditions and disasters may threaten the safety of life. In any weather condition, Synthetic Aperture Radars (SARs) ensure a good compromise of coverage and resolution thus letting to properly observe the phenomenon or area of interest.

When we speak about monitoring urban areas we usually think of monitoring buildings whose shape, in most cases, is that of a parallelepiped. In this paper, instead, our attention is given to big tanks, often present in the closest neighborhoods of urban centers, whose shape is that of a classical cylinder or that of a cylinder with a conic-shaped roof.

The interest for this kind of man-made structures arose for different reasons. Above all, the content of these tanks is often dangerous being easily flammable (fuel, hydrogen, other kind of gases). Hence the need of their surveillance especially in areas, like the African territories, where climate or vegetated areas make the propagation of fires easy. Moreover, differently from buildings which can greatly vary in terms of dimensions, shapes and materials from country to country and, sometimes, even from city to city in the same country, this kind of structure is everywhere the same: for safety and logistic reasons, the best way to store gases is in cylindrical tanks and these structures are everywhere adopted.

In this paper we are interested in investigating how the radar signal interacts with this kind of structures and how they appear in the SAR images. The aim is not only speculative but strongly applicative for the reasons above.

Up to now this kind of structures did not receive much consideration and nothing can be found in literature about it since the attention of scientific community was mostly devoted to understand and retrieve building structures in SAR images.

In Section 2 the approach we adopt is introduced while in Section 3 it is applied to a high resolution SAR image capturing different tanks in an Italian suburban area.

Comments on final results and future possible applications are given in the Conclusions. 


\section{SCATTERING MODEL FOR CYLINDER-LIKE STRUCTURES}

The approach we adopt to study these structures is based on the following consideration. Their shape can be, under certain circumstances, discretized and the cylinder reduced to a polyhedron. This possibility is interesting since the signal return from lateral surfaces may be treated and written like that backscattered from the building wall and ground modeled as a dihedral in [1]. Being the approach in [1] parametric and deterministic, its adaption for the case at issue will be too, and many applications may be derived, like the retrieval of features of interest for change detection, in the same way they have been derived for parallelepiped-like structures [2-4]. For example, some of these tanks present a floating roof whose height change with the volume content: in these cases, then, monitoring changes in the roof height means monitoring changes in the volume content.

Looking at Fig. 1 it is possible to qualitatively realize that, under some approximations, cylinders can be really dealt with as parallelepipeds. More precisely, some parts of the cylinder surface can be treated as the flat surface of the parallelepiped model in [1]. This is reasonable when the cylinder dimensions, namely the diameter $D$ and the height $h$, are much larger than the resolution cell dimensions, $\Delta \mathrm{x}$ and $\Delta y$, respectively in the azimuth and ground range direction. The 2D discretization process, suggested in Fig.1, shows a possible way to afford the matter: a grid is superimposed on the cylinder base and each peace of curve falling in a given resolution cell is approximated with the tangent at the curve. In 3D the cylinder is simply approximated by an appropriate irregular prism and, from the scattering point of view, each vertical face of the prism is treated as the building frontal wall in [1].

For the expressions of the scattered field we then refer to [1].
Obviously, also from the point of view of contributions succession in the range direction of the radar image, nothing changes when we discuss a parallelepiped or a cylinder. Indeed, adopting also for the cylinders the same models as in [1], layover, double reflection and shadow follow as expected, the only difference being in the curve borders of each region.

Conventional feature extraction applied to cylindrical shapes would use the layover or shadow range extensions to retrieve the cylinder height. This has been done also here to have a term of comparison.

In terms of tanks height extraction from double reflection contribution this implies to properly using the expressions in [1]. According to that approach, here the steps to follow for the tank height extraction are:

1.measuring and averaging the grey level of pixels of double reflection point located at the base of the cylinder;

2.relating this mean value to the radar cross section by means of calibration process;

3.inverting the expression relating the double reflection contribution to the radar cross section and the tank height (cfr [1] for mathematical expressions).

If we consider how a similar procedure works for buildings, now obvious modifications have to be introduced which are, essentially:

- in the extraction process: no more a line but a curve of double reflection has to be extracted;

- in the calibration procedure the average operations are to be performed on a smaller number of points.

A relevant scattering from all the border of the cylinder upper base (see Fig.2a) has been clearly recognized in each image (see Fig.2b). Even if the nature of this scattering is still under study, being clear from where it came, its extension along the range or azimuth direction has been used to retrieve the cylinder diameter with simple geometric expressions. More details about the extraction procedure will be given at the conference.
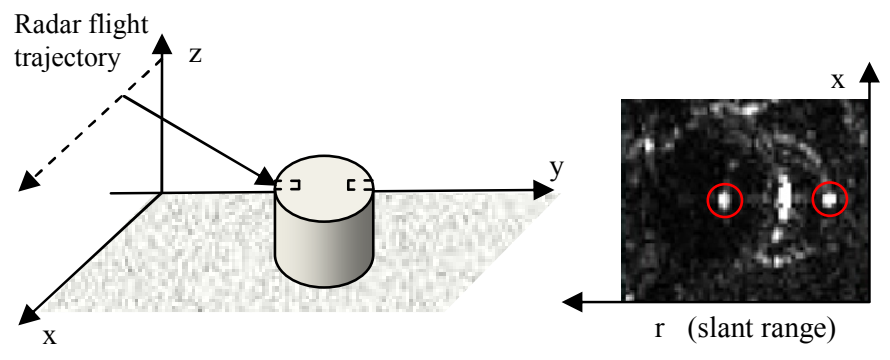

(a)

(b)

Figure 1

Figure 2 


\section{TANKS FEATURE EXTRACTION FROM SAR IMAGES}

The approach presented in the previous section has been applied to two different sites, in Algeria and Italy, see respectively Fig.3 and Fig.4, with different kind of products (from multi-look data to single look complex) and different resolutions (from $3 \mathrm{~m}$ in the stripmap mode to $1 \mathrm{~m}$ in the spotlight configuration) in order to extensively test the extraction procedure and also investigate the most suitable product for this task. This last issue is well dealt with in [5] and the reader is there referred for further details.

Here instead an example of application is shown to discuss the potentiality of our model-based approach when applied also to curved surfaces.

The data we worked at are a TerraSAR-X image, more precisely a Single Look Slant Range Complex (SSC) product which is, essentially, the basic single look product of the radar signal, given in complex representation. For more information about TerraSAR-X products see the document in [6].

Those data have been acquired on a suburban area of Naples, see Fig.4, where many tanks containing mainly fuel or dangerous acids are present.

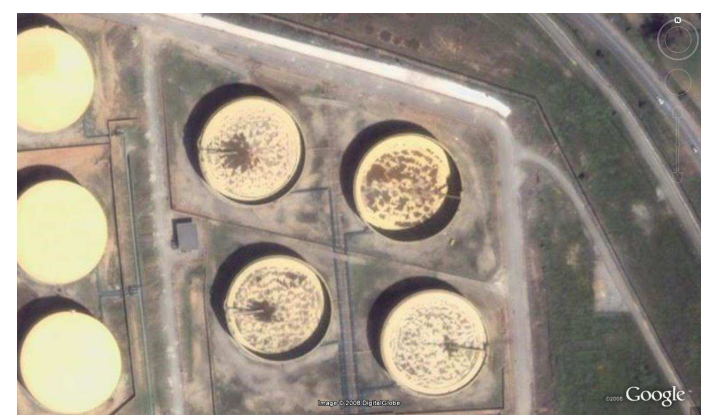

Figure 3

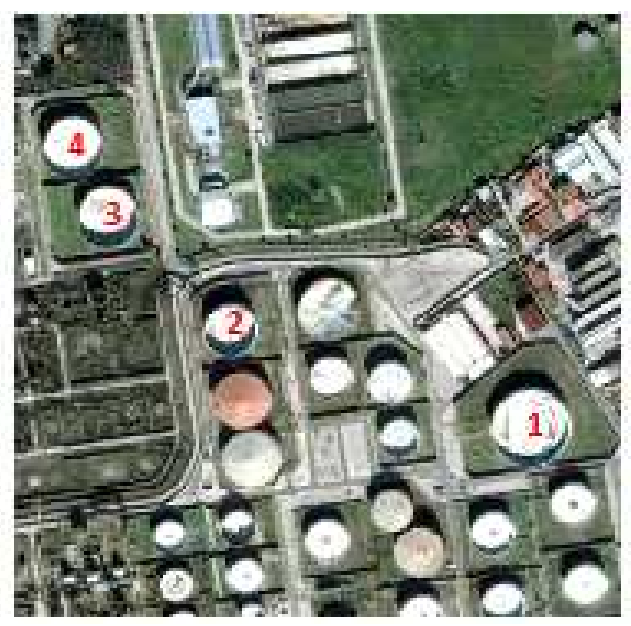

Figure 4
The site has been visited and the ground truth has been collected. Geometrical information about the tanks numbered in Fig. 4 has been also provided by the owner company.

The SSC product relevant to the scene in Fig.4 is shown in Fig.5. It has been acquired in X-band $(9.65 \mathrm{GHz}), \mathrm{HH}$ polarization, with a look angle of $54^{\circ}$ (scene centre) and, above all, in the spotlight mode giving in this case an image resolution of $1.10 \mathrm{~m}$ (azimuth) x $1.17 \mathrm{~m}$ (slant range).

As regards the tanks height, on this image as well as on the other kinds of products we worked in the conventional way retrieving the height from the layover extension obtaining the results in Table I, column 3, which present an error (column 4) smaller or in the order of the image resolution.

Studying the radiometry of the image, instead, and retrieving the height from the brightness of double reflection according to the new approach we proposed in [3] adapted here to curved surfaces we get the results in Table I, column 5 , which are really good and present an error (column 6) independent from the image geometric resolution as announced in [3]. Tanks $n .1$ and n. 4 have been used to radiometrically calibrate the SAR image.

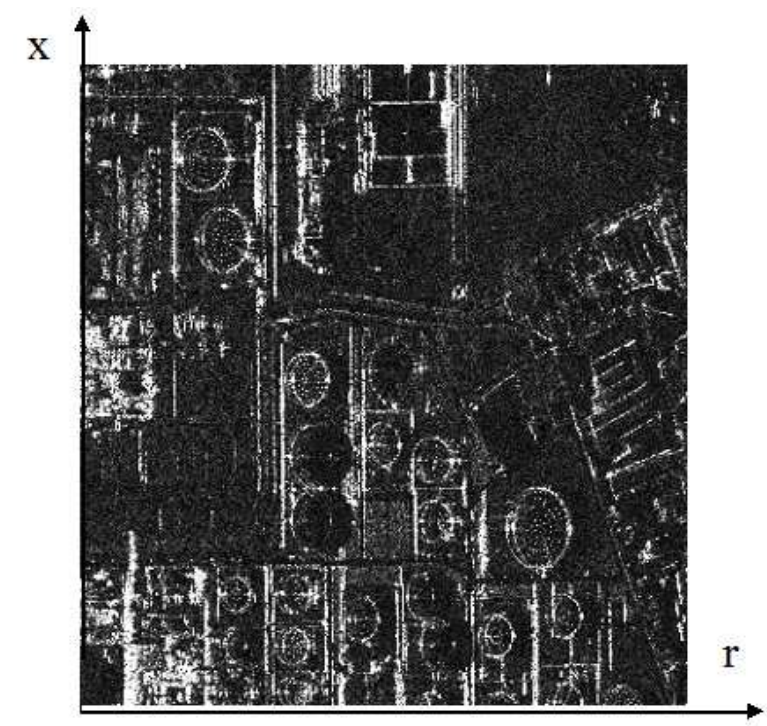

Figure 5

Table I

\begin{tabular}{|c|c|c|c|c|c|}
\hline N.Tank & $\begin{array}{c}\text { Height } \\
{[\mathrm{m}]} \\
\text { (ground } \\
\text { truth) }\end{array}$ & $\begin{array}{c}\text { Height }[\mathrm{m}] \\
\text { (from } \\
\text { geometry) }\end{array}$ & $\begin{array}{c}\left|\mathrm{e}_{\mathrm{h}}\right| \\
{[\mathrm{m}]}\end{array}$ & $\begin{array}{c}\text { Height }[\mathrm{m}] \\
\text { (from } \\
\text { radiometry) }\end{array}$ & $\begin{array}{c}\left|\mathrm{e}_{\mathrm{h}}\right| \\
{[\mathrm{m}]}\end{array}$ \\
\hline 1 & 14.40 & 12,37 & 2,03 & $\begin{array}{c}\text { NA } \\
\text { (calibrator) }\end{array}$ & - \\
\hline 2 & 14.60 & 15,46 & 0,86 & 14,35 & 0,25 \\
\hline 3 & 14.70 & 15,46 & 0,86 & 14,39 & 0,31 \\
\hline 4 & 14.70 & 12,37 & 2,33 & $\begin{array}{c}\text { NA } \\
\text { (calibrator) }\end{array}$ & - \\
\hline
\end{tabular}




\section{CONCLUSIONS}

In this paper the model introduced in [1] has been properly modified to study the scattering from isolated cylinders. The approach has been tested retrieving geometric features as done for buildings in [3]. In particular, the height extraction from double reflection contribution, which is one of the most interesting consequences of our model-based approach, is successful also in this case proving that the adaptation of the model to curved surfaces is feasible under the required conditions.

The often dangerous content of tanks represents a good reason to carefully monitoring areas with these structures. Moreover, the particularly simple geometry of tanks, closer to cylinders than a building to a parallelepiped, suggests that an unsupervised feature extraction algorithm has many chances of success and represents the next step of this work. A further idea to develop is based to the availability of an even better resolution, under 1 meter. Many tanks, indeed, are provided with a floating roof changing its position according to the volume content of the tank. In certain cases the position of the roof creates a further corner reflector and, consequently, another double reflection contribution from which brightness information about the volume content should be inferred.

Slightly different structures, like cylinders with conic-shaped roof are also under study.

\section{REFERENCES}

[1] G.Franceschetti, A.Iodice, D.Riccio, "A canonical problem in electromagnetic backscattering from buildings", IEEE Trans. Geosc. Remote Sensing, vol.40, pp.1787-1801, 2002.

[2] G.Franceschetti, R.Guida, A.Iodice, D.Riccio, "Extraction of Building Parameters from Single High Resolution SAR Images", IEEE Trans. Geosc. Remote Sensing, submitted.

[3] R.Guida, G.Franceschetti, A.Iodice, D.Riccio, G.Ruello, U.Stilla, "Building Feature Extraction via a Deterministic Approach: Application to Real High Resolution SAR Images", Proc. Geosc. Rem. Sens. Symp., pp.2681-2684, Barcelona (Spain), 2007.

[4] R. Guida, A. Iodice, D. Riccio, U. Stilla, "Model-Based Interpretation of High-Resolution SAR Images of Buildings", IEEE Journal of Selected Topics in Applied Earth Observations and Remote Sensing, vol.1, no.2, pp.107-119, June 2008.

[5] R. Guida, A. Iodice, D. Riccio," “Assessment of TerraSAR-X Products with a New Feature Extraction Application: Monitoring of Cylindrical Tanks", IEEE Trans. Geosc. Remote Sensing, in revision.

[6] T. Fritz, M. Eineder, J. Mittermayer, B. Schättler, W. Balzer, S. Buckreuß, R. Werninghaus, "TerraSAR-X Ground Segment Basic Product Specification Document", http://www.dlr.de/tsx/documentation, 2008. 